\title{
Efecto de las sales fundidas en la termofluencia del acero inoxidable tipo $304^{(*)}$
}

\author{
G. González-Rodríguez ${ }^{(*)}$, V. Salinas-Bravo ${ }_{(* *)}^{(*)}$ J. López-Huesca $^{(*)}$ \\ y A. Martínez-Villafañe ${ }^{(* *)}$
}

\begin{abstract}
Resumen Los problemas causados tanto por la corrosión por sales fundidas, así como por la termofluencia en los tubos de los sobrecalentadores y recalentadores de una planta de potencia que usa combustibles fósiles, reducen su vida prevista en diseño. La aceleración de la corrosión por sales fundidas es causada por la presencia de cenizas que contienen principalmente vanadio, sodio y azufre en la forma de $\mathrm{Na}_{2} \mathrm{SO}_{4}$ y $\mathrm{V}_{2} \mathrm{O}_{5}$, los cuales forman mezclas con eutécticos de bajo punto de fusión. Adicionalmente, los tubos están expuestos a la acción de altos esfuerzos y altas temperaturas, lo cual propicia la termofluencia del material. En este trabajo, se han realizado ensayos de termofluencia hasta la rotura en aire y en ambientes corrosivos en el rango de temperaturas de 620 a $660{ }^{\circ} \mathrm{C}$. Los ambientes corrosivos fueron $100 \% \mathrm{Na}_{2} \mathrm{SO}_{4}, 100 \% \mathrm{~V}_{2} \mathrm{O}_{5}$, y una mezcla $20 \% \mathrm{Na}_{2} \mathrm{SO}_{4}-80 \% \mathrm{~V}_{2} \mathrm{O}_{5}$.
\end{abstract}

Palabras clave: Corrrosión a temperatura elevada. Sales fundidas. Termofluencia. Sobrecalentador. Recalentador. Vida residual.

\section{Effect of molten salts on the creep resistance of 304 Type stainless steel}

\begin{abstract}
Problems caused by both hot corrosion and creep type-damage occuring on superheater and reheater tubes of power plants using heavy oil as fuel shorten their design lives. The acceleration of hot corrosion attack of boilers is caused by the presence of fuel ash deposits containing mainly vanadium, sodium and sulphur, in the form of $\mathrm{Na}_{2} \mathrm{SO}_{4}$ and $\mathrm{V}_{2} \mathrm{O}_{5}$ which form low melting point compounds. In addition to this, the tubes are exposed to the action of both high stresses and high temperatures, producing the so called creep damage. In this work, creep rupture tests were carried out in the temperature range of 620 to $660{ }^{\circ} \mathrm{C}$ in static air and in corrosive environments. The corrosive environments included $100 \% \mathrm{Na}_{2} \mathrm{SO}_{4}, 100 \% \mathrm{~V}_{2} \mathrm{O}_{5}$ and a $80 \% \mathrm{~V}_{2} \mathrm{O}_{5}+20 \% \mathrm{Na}_{2} \mathrm{SO}_{4}$ mixture.
\end{abstract}

Keywords: Hot corrosion. Molten salts. Creep. Superheater. Reheater. Residual life.

\section{INTRODUCCIÓN}

Los componentes usados en intercambiadores de calor, especialmente sobrecalentadores $(\mathrm{SH})$ y recalentadores (RH) de un generador de vapor, están sujetos a la acción conjunta de ambientes corrosivos y esfuerzos mecánicos a altas temperaturas, lo cual produce por una parte corrosión, y por otra termofluencia de los materiales. Los elementos metálicos del SH y del RH son diseñados para resistir la termofluencia durante un determinado período, éste

(•) Trabajo recibido el día 7 de julio de 1994

(*) Instituto de Investigaciones Eléctricas. Dpto. de Fisicoquímica Aplicada. Apartado postal 475. 62000Cuernavaca. Mor., México.

(**) Centro de Investigaciones en Materiales Avanzados. León Tolstoi, 116, Complejo Industrial Chihuahua, Chihuahua, Chih., México. generalmente no se cumple debido al efecto de la corrosión. Por ejemplo, Nicholls (1) encontró que los tiempos de rotura y la elongación a la rotura para el Nimonic 108 se redujeron hasta siete veces respecto a los datos obtenidos en aire cuando los experimentos se realizaron en $50 \% \mathrm{Na}_{2} \mathrm{SO}_{4}+50 \%$ $\mathrm{NaCl}$ a $800{ }^{\circ} \mathrm{C}$. Resultados similares se obtuvieron para el acero inoxidable 316. Similarmente, Suryanarayanan (2) usó el acero 304 en $100 \% \mathrm{Na}_{2} \mathrm{SO}_{4} \mathrm{y}$ en $75 \% \mathrm{Na}_{2} \mathrm{SO}_{4}+25 \% \mathrm{NaCl}$ en el rango de temperaturas de 600 a $700{ }^{\circ} \mathrm{C}$, encontrando un efecto despreciable a $600{ }^{\circ} \mathrm{C}$ con el $\mathrm{Na}_{2} \mathrm{SO}_{4}$, debido a que éste no fundía, mientras que a 650 y $700{ }^{\circ} \mathrm{C}$ encontró también que los tiempos de rotura fueron menores y las velocidades de deformación mayores en estos ambientes en comparación con los resultados obtenidos en aire, debido a la formación de compuestos con eutécticos con bajos puntos de fusión, 
como el Ni-Ni ${ }_{3} \mathrm{~S}_{2}$ y el $\mathrm{Na}_{2} \mathrm{SO}_{4}$. Oh y Park (3) también llevaron a cabo estudios de termofluencia del acero inoxidable 304 usando primero una capa interna de $85 \% \quad \mathrm{~V}_{2} \mathrm{O}_{5}+10 \% \quad \mathrm{Na}_{2} \mathrm{SO}_{4}+5 \%$ $\mathrm{Fe}_{2} \mathrm{O}_{3}$ y una capa externa de $10 \% \quad \mathrm{~V}_{2} \mathrm{O}_{5}+85 \%$ $\mathrm{Na}_{2} \mathrm{SO}_{4}+5 \% \quad \mathrm{Fe}_{2} \mathrm{O}_{3}$ en el rango de temperaturas de 603 a $750{ }^{\circ} \mathrm{C}$, obteniendo que, en especial a 630 ${ }^{\circ} \mathrm{C}$, el tiempo de rotura en ambiente corrosivo mostró una considerable disminución con respecto a los tiempos obtenidos en aire, sugiriendo que los productos de corrosión con bajo punto de fusión tienen un efecto más severo sobre la vida de los materiales. Similares resultados se han obtenido para el Inconel 601 en $\mathrm{NaVO}_{3}$ y en $85 \% \mathrm{NaVO}_{3}+15 \%$ $\mathrm{Na}_{2} \mathrm{SO}_{4}$ a 650,700 y $750{ }^{\circ} \mathrm{C}(4)$, para el acero $2,25 \mathrm{Cr}-1 \mathrm{Mo}$ en $60 \% \mathrm{~K}_{2} \mathrm{SO}_{4}+40 \% \mathrm{NaCl}$ a 550 , 600 y $650{ }^{\circ} \mathrm{C}(5)$, así como para el acero inoxidable 304, el Inconel 718 e Inconel 751 en $90 \% \mathrm{Na}_{2} \mathrm{SO}_{4}$ $+10 \% \mathrm{NaCl}$ a $800^{\circ} \mathrm{C}(6)$. Algunos de los mecanismos propuestos, por los que los depósitos corrosivos disminuyen las propiedades mecánicas, son la reducción en la sección de trabajo (7), producción de huecos en los límites de grano por la difusión hacia fuera de cationes metálicos (1), y formación de eutécticos con bajos puntos de fusión acelerando la velocidad de difusión de las especies corrosivas que conducen a una penetración intergranular en toda la sección de trabajo del metal (2).

Por otra parte, el combustible usado en calderas, que es un derivado del petróleo, contiene impurezas como vanadio, sodio y azufre, las cuales, durante la combustión, forman compuestos de $\mathrm{Na}_{2} \mathrm{SO}_{4}$ y $\mathrm{V}_{2} \mathrm{O}_{5}$ principalmente. Adicionalmente, el combustible puede contaminarse durante el transporte o almacenamiento en ambientes marinos con $\mathrm{NaCl}$, propiciando la formación de compuestos de aún más bajos puntos de fusión, reduciendo el umbral de temperatura a partir del cual se inicia en servicio la corrosión por sales fundidas, que puede acortar sustancialmente la vida útil de los materiales (8 y 9). La corrosión por sales fundidas es un fenómeno de mucha importancia, no sólo en tubos de calderas sino, también, en otros equipos que operan a alta temperatura como turbinas de gas, máquinas diesel, máquinas de aviones, etc. Normalmente, la corrosión por sales fundidas y las propiedades mecánicas en alta temperatura han sido estudiadas separadamente, y los componentes que operan a alta temperatura han sido escogidos tomando como base sus propiedades mecánicas o su resistencia a la corrosión. Sin embargo, en la práctica, el material estará sujeto a la acción conjunta de ambos fenómenos. Así, el objetivo de este artículo es tratar de predecir la vida residual de uno de los materiales más comúnmente usados en tubos de sobrecalentadores o recalentadores, el acero inoxidable tipo ASTM SA 213 TP 304H, en presencia de ambientes corrosivos sintéticos, simulando los encontrados en una caldera.

\section{EXPERIMENTACIÓN}

El material usado en este trabajo fue el acero inoxidable tipo ASTM SA 213 TP 304H cuya composición química se da en la tabla I. A partir de tubos nuevos, se tomaron probetas planas de 30,0 $\mathrm{mm}$ de longitud y una sección de $2,0 \times 3,2 \mathrm{~mm}^{2}$ en el área de trabajo. Antes de los ensayos de termofluencia, se eliminaron las huellas del maquinado en las probetas, con lijas grado 600 , y se cubrieron uniformemente con $500 \mathrm{mg} / \mathrm{cm}^{2}$ de depósito, el cual se había preparado previamente usando agua destilada para formar una pasta. Los depósitos que se utilizaron fueron $100 \% \mathrm{Na}_{2} \mathrm{SO}_{4}, 100 \% \mathrm{~V}_{2} \mathrm{O}_{5}$ y una mezcla de $80 \% \mathrm{~V}_{2} \mathrm{O}_{5}+20 \% \mathrm{Na}_{2} \mathrm{SO}_{4}$, la cual es la mezcla más corrosiva para los aceros inoxidables de la serie 300 (10). Los ensayos de termofluencia se realizaron a esfuerzo constante en una máquina de termofluencia con un brazo de palanca con una relación 8:1 a las temperaturas de 620,640 y $660{ }^{\circ} \mathrm{C}$. La temperatura a lo largo del eje de la probeta se monitoreó con un termopar Cromel-Alumel con lo que se detectó que las fluctuaciones de la temperatura durante el ensayo eran de $\pm 2{ }^{\circ} \mathrm{C}$. Después de fracturadas, las probetas se encapsulaban en baquelita para observarlas en el microscopio óptico o electrónico, y los productos de corrosión se analizaron por medio de difracción de rayos $\mathrm{X}$.

\section{RESULTADOS}

La figura 1 muestra la variación del tiempo de rotura, $t_{\mathrm{r}}$, en función del esfuerzo aplicado, $\sigma$, en escala doble logarítmica para el acero 304 en los distintos ambientes a 620,640 y $660{ }^{\circ} \mathrm{C}$. Se puede ver claramente que los tiempos de rotura más cortos se obtuvieron en la mezcla de $80 \% \mathrm{~V}_{2} \mathrm{O}_{5}+20 \%$

TABLA I.- Composición química del acero 304, \% en masa

TABLE I.-Chemical composition of 304 type stainless steel, \% wt.

\begin{tabular}{|c|c|c|c|c|c|c|c|}
\hline $\mathrm{S}$ & $\mathrm{C}$ & $\mathrm{Si}$ & $\mathrm{Mn}$ & $\mathrm{Mo}$ & $\mathrm{Ni}$ & $\mathrm{Cr}$ & $\mathrm{Fe}$ \\
\hline 0,005 & 0,068 & 0,90 & 1,40 & 0,21 & 8,80 & 18,15 & Resto \\
\hline
\end{tabular}




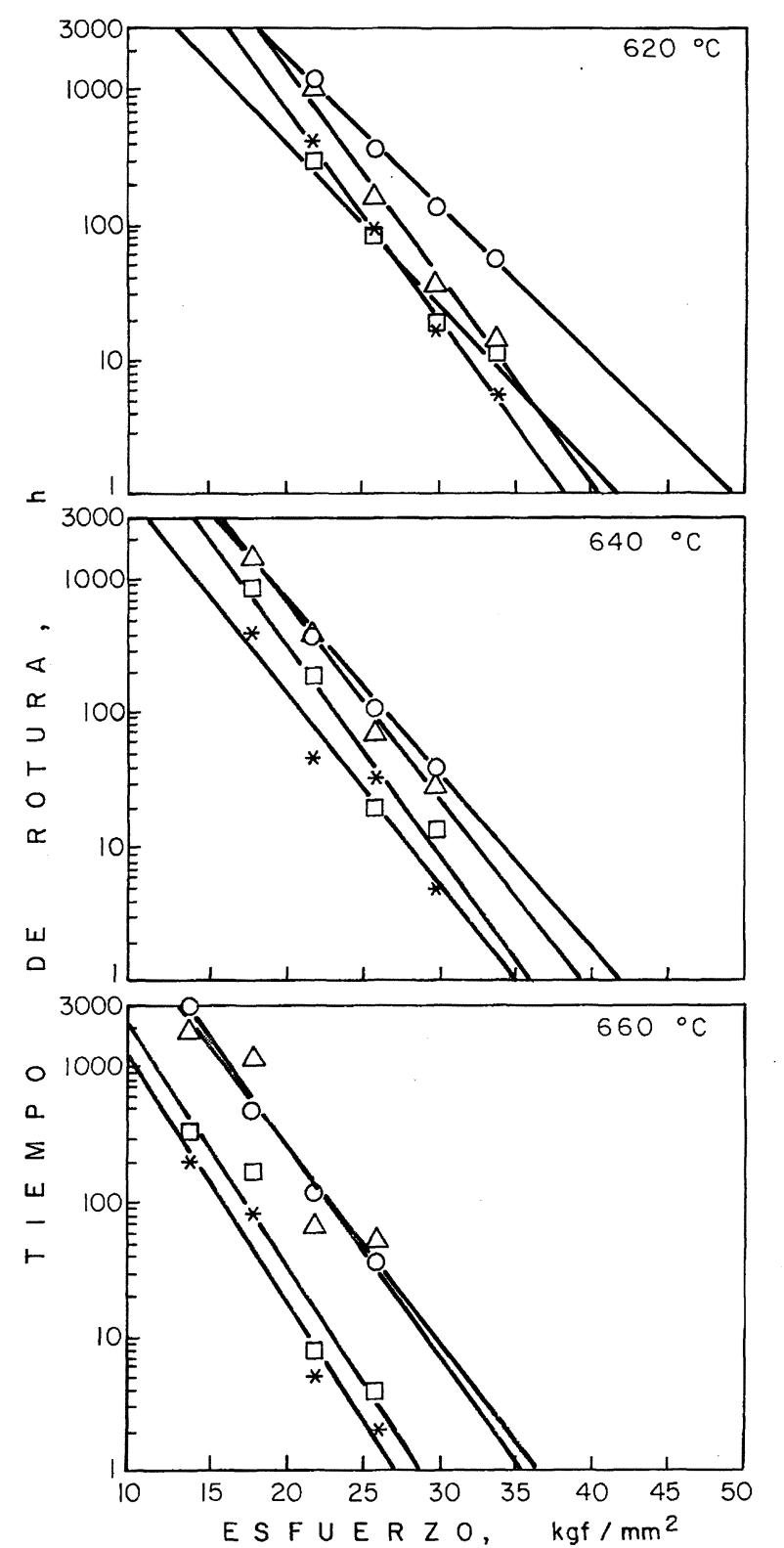

O Aire; $\triangle \mathrm{Na}_{2} \mathrm{SO}_{4} ; \square \mathrm{V}_{2} \mathrm{O}_{5} ; * 80 \% \mathrm{~V}_{2} \mathrm{O}_{5}+20 \% \mathrm{Na}_{2} \mathrm{SO}_{4}$

FIG. 1.- Variación del tiempo de rotura en función del esfuerzo aplicado en distintos ambientes a 620 , 640 y $660{ }^{\circ} \mathrm{C}$.

FIG. 1.-Relation between the rupture time and the applied stress in different environments at 620, 640 and $660^{\circ} \mathrm{C}$.

$\mathrm{Na}_{2} \mathrm{SO}_{4}$, y el menos agresivo fue el $\mathrm{Na}_{2} \mathrm{SO}_{4}$, mientras que la figura 2 muestra la variación entre el tiempo de rotura y el esfuerzo aplicado en escala doble logarítmica a diferentes temperaturas para el $\mathrm{Na}_{2} \mathrm{SO}_{4}, \mathrm{~V}_{2} \mathrm{O}_{5}$ y la mezcla $80 \% \mathrm{~V}_{2} \mathrm{O}_{5}+20 \%$ $\mathrm{Na}_{2} \mathrm{SO}_{4}$. Tanto en la figura 1 como en la 2 es obvia la relación lineal entre $\log \left(t_{\mathrm{r}}\right)$ y $\log (\sigma)$ indicando que una función de la forma $t_{\mathrm{r}}=A \sigma^{\mathrm{n}} \exp (Q / k T)$ gobierna la relación. Los valores de las constantes $A$ y $n$, así como de la energía de activación, $Q$, que se encontraron para cada uno de los depósitos, die-

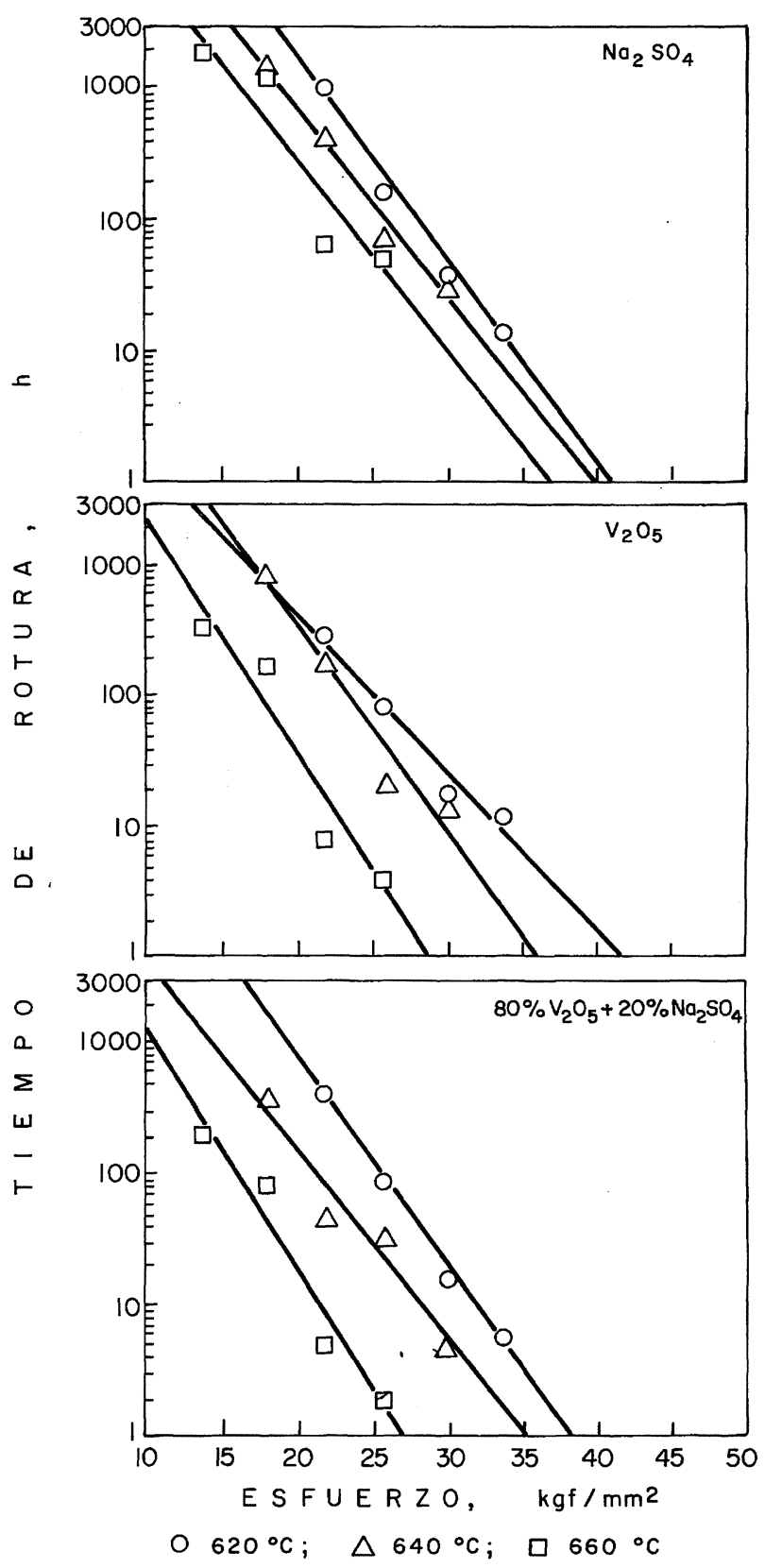

FIG. 2.- Variación del tiempo de rotura con el esfuerzo aplicado a distintas temperaturas en $\mathrm{Na}_{2} \mathrm{SO}_{4}, \mathrm{~V}_{2} \mathrm{O}_{5}$ y la mezcla $80 \% \mathrm{~V}_{2} \mathrm{O}_{5}+20 \%$ $\mathrm{Na}_{2} \mathrm{SO}_{4}$.

FIG. 2.- Relation between the rupture time and the applied stress at different temperatures in $\mathrm{Na}_{2} \mathrm{SO}_{4}$, $\mathrm{V}_{2} \mathrm{O}_{5}$, and the mixture $80 \% \mathrm{~V}_{2} \mathrm{O}_{5}+20 \% \mathrm{Na}_{2} \mathrm{SO}_{4}$.

ron factores de correlación de $0,93,0,95$ y 0,90 para el $\mathrm{Na}_{2} \mathrm{SO}_{4}, \mathrm{~V}_{2} \mathrm{O}_{5}$ y mezcla, respectivamente. El modo de fractura fue, en todos los casos, intergranular, como se puede apreciar en la figura 3 , con la diferencia de que en las pruebas en aire, la mayoría de las grietas se presentaban en el interior de la muestra, mientras que en las muestras fracturadas en depósitos, las grietas se presentaban en la superficie, siendo notable, asimismo, la presencia de microcavidades en los límites de grano. 

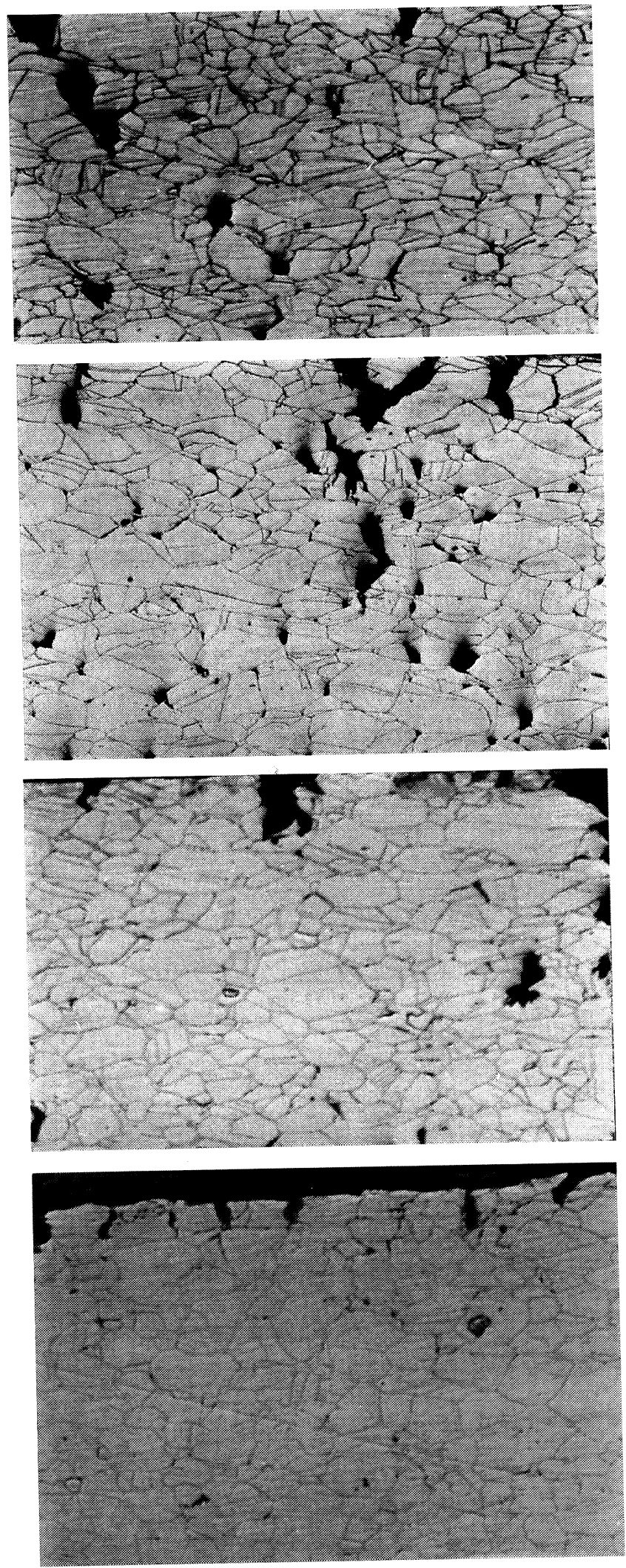

FIG. 3.- Micrografías del acero 304 fracturado en: a) Aire, b) $100 \% \mathrm{Na}_{2} \mathrm{SO}_{4}$, c) $100 \% \mathrm{~V}_{2} \mathrm{O}_{5} \mathrm{y}$ d) $80 \% \mathrm{~V}_{2} \mathrm{O}_{5}+20 \% \mathrm{Na}_{2} \mathrm{SO}_{4} \cdot \times 200$.

FIG. 3.- Photomicrographs of 304 type stainless steel fractured in a) Air, b) $\left.100 \% \mathrm{Na}_{2} \mathrm{SO}_{4}, c\right) 100$ $\% \mathrm{~V}_{2} \mathrm{O}_{5}$ and d) $80 \% \mathrm{~V}_{2} \mathrm{O}_{5}+20 \% \mathrm{Na}_{2} \mathrm{SO}_{4} \times 200$.
La figura 4 muestra el efecto de la temperatura sobre el porcentaje de reducción en área de las probetas al finalizar la prueba para todos los medios estudiados. Se puede ver que los tres depósitos causaron una mayor reducción en el área de trabajo, comparados con los resultados en aire, lo cual se debe a la agresividad de cada uno de los depósitos. Para las muestras fracturadas en $100 \% \mathrm{~V}_{2} \mathrm{O}_{5}$ ó en la mezcla $80 \% \mathrm{~V}_{2} \mathrm{O}_{5}+20 \% \mathrm{Na}_{2} \mathrm{SO}_{4}$ se logró detectar la presencia de vanadio dentro de una grieta por medio del análisis de dispersión de rayos X. Sin embargo, para las muestras fracturadas en $100 \%$ $\mathrm{Na}_{2} \mathrm{SO}_{4}$ o en la mezcla, no se detectó la presencia de sodio, lo que se muestra en la figura 5 , mientras que en la figura 6 se muestra la distribución de vanadio, cromo y hierro dentro de la grieta. Se puede ver cómo el vanadio penetra intergranularmente mientras que la presencia de hierro y cromo muestra la remoción de los elementos aleantes del acero. Finalmente, los estudios de difracción de rayos $\mathrm{X}$ revelaron la presencia de compuestos formados sobre la superficie dẹ la muestra una vez que ésta ha sido fracturada, cuya relación se incluye en la tabla II.

\section{DISCUSIÓN}

Los resultados del presente trabajo muestran que ninguno de los mecanismos de falla por la interacción corrosión por sales fundidas y termofluencia, indicados en la literatura, explican por sí solos el acelerado daño de los materiales. Así, se mostró que, efectivamente, hay disminución de la sección de trabajo de la muestra producida por la corrosión, que existe la formación de huecos o microcavidades en los límites de grano disminuyendo la cohesión entre los granos, así como la remoción de elemen-

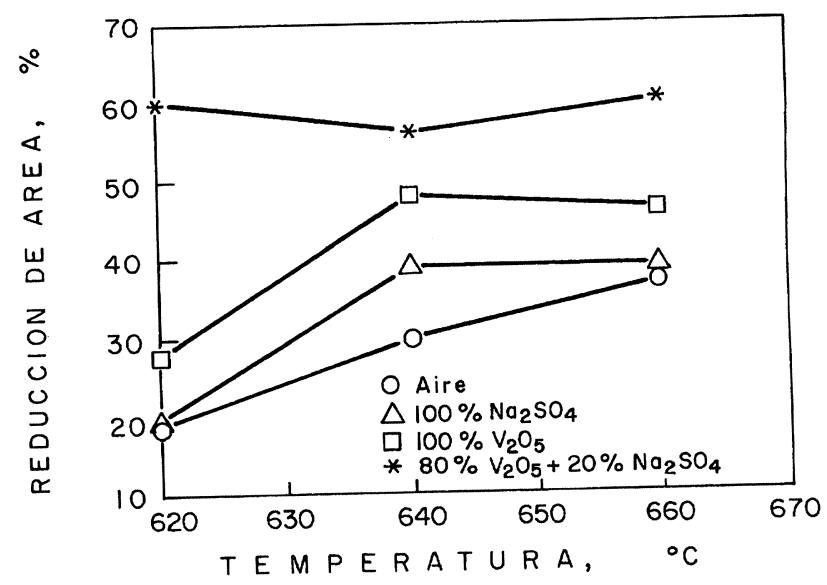

FIG. 4.- Efecto de la temperatura sobre la reducción en área en los distintos ambientes usados.

FIG. 4.- Variation of percentage reduction in area with temperature for the different environments. 
TABLA II.- Compuestos identificados por difracción de rayos $\mathrm{X}$, debido a la reacción del acero 304 con los distintos depósitos estudiados

TABLE II.- List of compounds identified from the $X$-ray analysis of corrosion products of the creepplus-hot corroded samples

\begin{tabular}{|c|l|l|c|}
\hline Depósito & $\mathrm{Na}_{2} \mathrm{SO}_{4}$ & $\mathrm{~V}_{2} \mathrm{O}_{5}$ & $\mathrm{~V}_{2} \mathrm{O}_{5}-\mathrm{Na}_{2} \mathrm{SO}_{4}$ \\
\hline Compuesto & $\mathrm{Fe}_{2} \mathrm{O}_{3}$ & $\mathrm{Fe}_{2} \mathrm{O}_{3}$ & $\mathrm{Fe}_{2} \mathrm{O}_{3}$ \\
& $\mathrm{Cr}_{2} \mathrm{Fe}_{2} \mathrm{O}_{3}$ & $\mathrm{FeVO}_{4}$ & $\mathrm{FeV}_{2} \mathrm{O}_{4}$ \\
& $\mathrm{Na}_{2} \mathrm{SO}_{4}$ & $\mathrm{Cr}_{2} \mathrm{O}_{3}$ & $\mathrm{FeS}_{2}$ \\
& $\mathrm{Cr}_{2} \mathrm{O}_{3}$ & $\mathrm{NiO}$ & $\mathrm{MnSiO}_{3}$ \\
& $\mathrm{NiO}$ & & $\mathrm{Cr}_{2} \mathrm{O}_{3}$ \\
& & & $\mathrm{NiO}$ \\
\hline
\end{tabular}
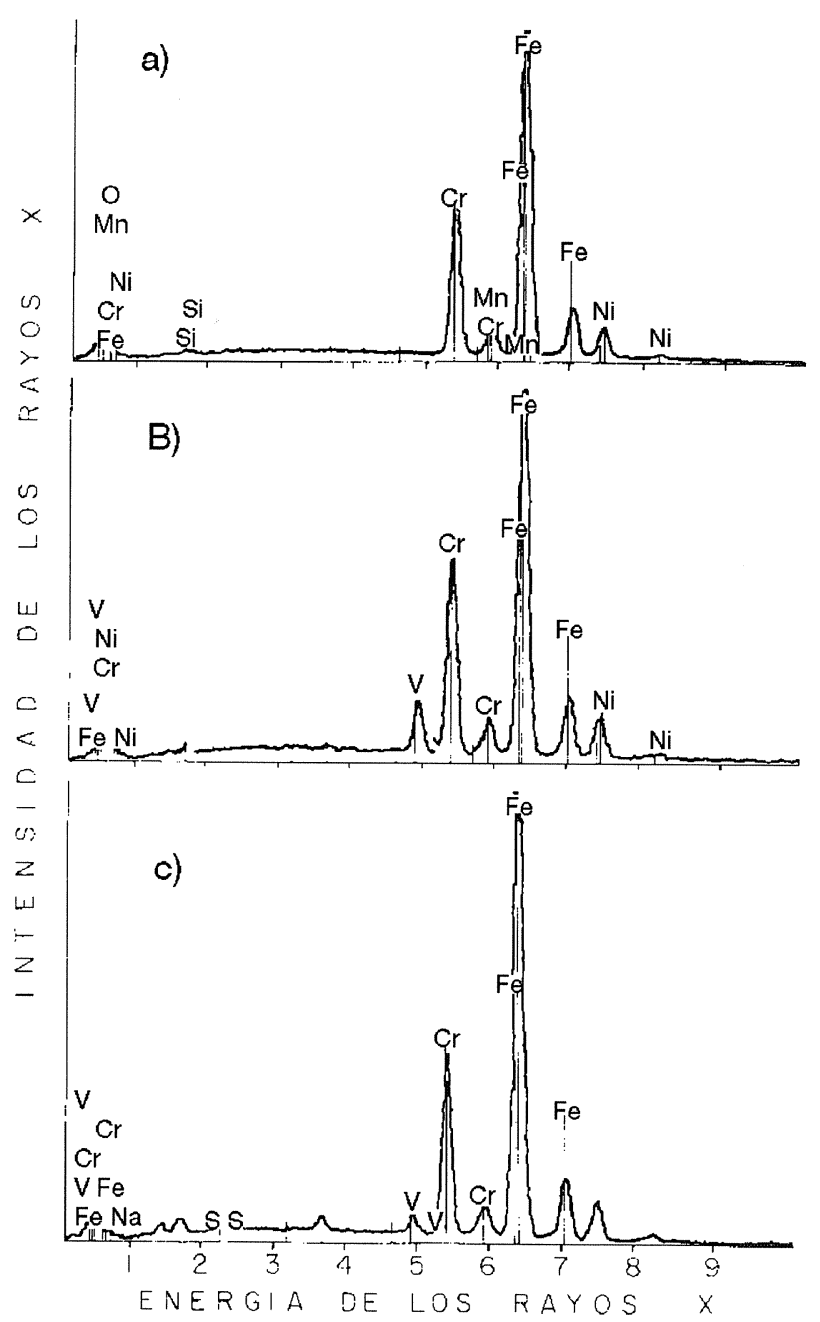

FIG. 5.- Espectros de dispersión de rayos X realizados en el interior de una grieta de una muestra fracturada en: a) $100 \% \mathrm{Na}_{2} \mathrm{SO}_{4}$, b) $100 \% \mathrm{~V}_{2} \mathrm{O}_{5}$ y c) $80 \% \mathrm{~V}_{2} \mathrm{O}_{5}+20 \% \mathrm{Na}_{2} \mathrm{SO}_{4}$.

FIG. 5.-X-ray pattern done inside a crack of a specimen fractured in a) $\left.100 \% \mathrm{Na}_{2} \mathrm{SO}_{4}, b\right) 100 \%$

$\mathrm{V}_{2} \mathrm{O}_{5}$ and c) $80 \% \mathrm{~V}_{2} \mathrm{O}_{5}+20 \% \mathrm{Na}_{2} \mathrm{SO}_{4}$.
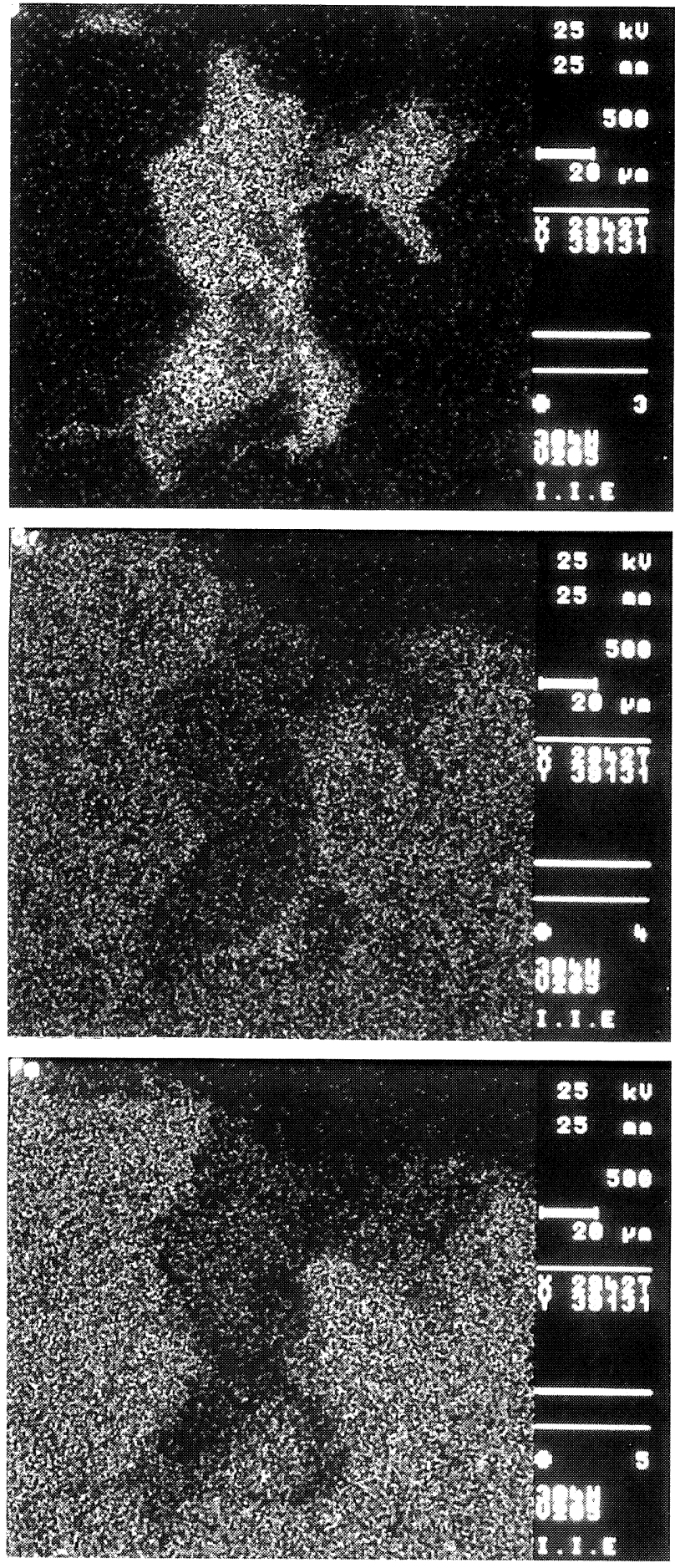

FIG. 6.- Micrografías mostrando la distribución de vanadio, cromo y hierro en el interior de una grieta de una muestra fracturada en $100 \% \mathrm{~V}_{2} \mathrm{O}_{5}$.

FIG. 6.- Mapping of vanadium, chromium, and iron inside a crack of a specimen fractured in $100 \% \mathrm{~V}_{2} \mathrm{O}_{5}$.

tos aleantes por las sales fundidas disminuyendo sus propiedades mecánicas como de su resistencia a la corrosión. El daño por termofluencia está relacionado con el punto de fusión de los compuestos corrosivos. El sulfato de sodio tiene un punto de 
fusión de $884{ }^{\circ} \mathrm{C}$, el cual está fuera del rango de temperaturas empleadas en este trabajo, mientras que el pentóxido de vanadio funde a los $660^{\circ} \mathrm{C}$ y la mezcla usada aquí lo hace a los $615^{\circ} \mathrm{C}$, valores que están dentro del rango de temperaturas empleadas. Esto explica el poco efecto del sulfato de sodio sobre el tiempo de rotura, el gran efecto de la mezcla y el efecto intermedio del pentóxido de vanadio. Estudios realizados por Ahila (5) demostraron que los sistemas ternarios $\mathrm{V}_{2} \mathrm{O}_{5}-\mathrm{NiO}-\mathrm{Cr}_{2} \mathrm{O}_{3}$ pueden estar en estado líquido a temperaturas tan bajas como los $550{ }^{\circ} \mathrm{C}$. Un eutéctico similar se observa en el sistema $\mathrm{Fe}_{2} \mathrm{O}_{3}-\mathrm{V}_{2} \mathrm{O}_{5}-\mathrm{Cr}_{2} \mathrm{O}_{3}$, el cual tiene un punto de fusión todavía más bajo, $480^{\circ} \mathrm{C}$. Los estudios de difracción de rayos $\mathrm{X}$ de este trabajo mostraron la presencia de compuestos tales como NiO, $\mathrm{Cr}_{2} \mathrm{O}_{3}, \mathrm{Fe}_{2} \mathrm{O}_{3}$ y los vanadatos de hierro $\mathrm{FeVO}_{4}$ y $\mathrm{FeV}_{2} \mathrm{O}_{4}$. En determinadas circunstancias, se pueden formar óxidos porosos no protectores debido a la formación de mezclas eutécticas de óxidos, como en el caso del $\mathrm{V}_{2} \mathrm{O}_{5}$. Al adicionar sulfato de sodio al pentóxido de vanadio, el vanadio reacciona con el hierro para formar vanadatos $\left(\mathrm{FeVO}_{4}, \mathrm{FeV}_{2} \mathrm{O}_{4}\right)$, mientras que el azufre reacciona con el hierro para formar sulfuros como el $\mathrm{FeS}_{2}$ y $\mathrm{Ni}_{3} \mathrm{~S}_{2}$, los cuales fueron detectados por rayos $\mathrm{X}$ teniendo el $\mathrm{Ni}_{3} \mathrm{~S}_{2}$ un punto de fusión de $635^{\circ} \mathrm{C}$. Esta fase líquida penetra a través de los límites de grano hasta alcanzar las microcavidades producidas durante la termofluencia, aumentando así la acumulación del daño por ésta y la pérdida de sección transversal producto de la corrosión. Hubo otros compuestos que contienen elementos aleantes del acero como cromo, níquel y hierro. La remoción de estos elementos aleantes podría hacer que disminuyesen las propiedades mecánicas y la resistencia a la corrosión del acero haciéndolo muy susceptible al fallo, aun cuando para apoyar esta aseveración se requiere una ampliación del trabajo.

\section{CONCLUSIONES}

Los resultados de este trabajo sobre el efecto de las sales fundidas en la resistencia a la termofluencia del acero inoxidable tipo 304 permiten extraer las siguientes conclusiones:

- Comparados con los resultados en aire, y dentro del rango de temperaturas estudiado, la mezcla $80 \% \mathrm{~V}_{2} \mathrm{O}_{5}+20 \% \mathrm{Na}_{2} \mathrm{SO}_{4}$ fue la sal que más redujo los tiempos de rotura así como en la que se obtuvieron las mayores reducciones en área. Por su parte, el $\mathrm{V}_{2} \mathrm{O}_{5}$ tuvo un efecto menos agresivo, debido a la formación de compuestos con eutécticos de bajos puntos de fusión como el $\mathrm{FeVO}_{4}, \mathrm{FeV}_{2} \mathrm{O}_{4}, \mathrm{~V}_{2} \mathrm{O}_{5}-\mathrm{Cr}_{2} \mathrm{O}_{3}-\mathrm{NiO}, \mathrm{Fe}_{2}-\mathrm{O}_{3}-\mathrm{NiO}$, $\mathrm{FeS}_{2}$ y $\mathrm{Ni}_{3} \mathrm{~S}_{2}$.

- Los eutécticos tienen puntos de fusión que caen dentro del intervalo de temperaturas estudiado destruyen los óxidos protectores del material, disminuyen la sección de trabajo de las muestras y atacan los límites de grano del material ya debilitados por la aparición de las microcavidades intergranulares, produciendo fallas prematuras en el material.

- Los resultados obtenidos en $\mathrm{Na}_{2} \mathrm{SO}_{4}$ fueron virtualmente idénticos a los obtenidos en aire, debido a que su punto de fusión, $884^{\circ} \mathrm{C}$, está muy por encima de las temperaturas usadas aquí, sin dar lugar a procesos de corrosión, y propiciando que el material fallara por efectos mecánicos.

\section{REFERENCIAS}

(1) Nicholls, J.R., Samuel, J., Hurst, R.C. y Hancock, P. Behaviour of High Temperature Alloys in Aggressive Environments. Proc. Intern. Conf. Petten. The Metals Society. Países Bajos, 1979: 911-926.

(2) Suryanarayanan, V., IYeR, K.J.L. y RAdHAKRISHNAN, V.M. Mater. Sci. Eng. A112, 1989: 107-116.

(3) OH, S.H y PARK, I.S. 8th Intern. Conf. on Offshore Mechanics and Artic Engineering. La Haya (Países Bajos), 1994: 187-191.

(4) Swaminathan, J., Saghavan, A. Mater. High Temp., 10 (4), 1992: 242-250.

(5) Ahila, S., RAmakrishna, S. y Radhakrishnan, V.M. Trans. Indian Inst. Met., 46 (4), 1993: 215-223.

(6) Yoshiba, M. y Miyagawa, O. Intern. Conf. on Creep. JSME, IMechE, ASME, ASTM. Tokio (Japón) 1986: 193-198.

(7) Kofstad, P. Proc. Symp. on High Temperature Corrosion in Coal Gasification Atmosphere. J.F.Norton (ed.), Elsevier, Londres, 1984: 207-222.

(8) Stringer, J. High Temp. Technol., 3 (3), 1985: 119-141.

(9) JONES, R.L. High Temp. Technol., 6 (4), 1988: 187-193.

(10) Wong, A., Mariaca, L. y Peraza, A. IX Inter-American Conference on Materials Technology. OEA. Santiago de Chile, 1987: 339-343. 